\title{
Quadrilateral finite element Monte Carlo simulation of complex shape compound FETs
}

\author{
S. BABIKER, A. ASENOV, J. R. BARKER and S. P. BEAUMONT \\ Nanoelectronics Research Centre, Department of Electronics and Electrical Engineering \\ University of Glasgow, Glasgow, G12-8QQ, UK.
}

\begin{abstract}
The complex recess and gate shape of modern compound FETs greatly affect the device parasitics and therefore impose the need for proper description of the device geometry and surface conditions in any practical device simulations. In this paper we describe a new Monte Carlo (MC) module incorporated in our Heterojunction 2D Finite element FET simulator H2F [1]. The module combines realistic quadrilateral finite-element description of the device geometry with realistic particle simulation of the non-equilibrium hot carrier transport in short recess gate compound FETs. A Single Programme Multiple Data (SPMD) parallel approach makes it possible to use our MC simulator for practical design work, generating the necessary I-V characteristics in parallel. The capabilities of the finite element MC module are illustrated in example simulations of a $200 \mathrm{~nm}$ pseudomorphic HEMT fabricated in the Nanoelectronics Research Centre of Glasgow University.
\end{abstract}

Keywords: Finite element, isoparametric mapping, plasma oscillations

\section{INTRODUCTION}

Most modern sub micrometer compound FETs are single or double recess devices with complicated recess geometries. In some state of the art $100 \mathrm{~nm}$ gate length FETs the length of the recess region is comparable to the length of the gate. The device parasitics are strongly affected by the shape and surface conditions of the recess. These parasitics are critical to the device performance and it is therefore important that the Monte-Carlo simulators should allow for a precise description of the device geometry and proper handling of the surface effects. The use of finite elements as a discretization approach offers the necessary flexibility. Most MC simulators consider oversimplified planar or rectangular solution domains to represent the simulated compound FET. In addi- tion, the surface potential pinning effects are normally neglected.

In this paper we report on a new Monte Carlo (MC) module incorporated in our Heterojunction 2D Finite element FET simulator H2F [1] and based on quadrilateral finite elements. The capabilities of the new finite element MC module are illustrated in example simulations of a $200 \mathrm{~nm}$ gate length pseudomorphic HEMT fabricated in the Nanoelectronics Research Centre of Glasgow University.

\section{THE MONTE CARLO MODULE}

The Galerkin finite element approach has been adopted to solve Poisson's equation. 


$$
\frac{\partial}{\partial x} \varepsilon \frac{\partial}{\partial x} \Psi+\frac{\partial}{\partial y} \varepsilon \frac{\partial}{\partial y} \Psi+\rho_{B}+\rho_{I}=0
$$

where $\rho_{B}$ is the bulk charge density and $\rho_{I}$ is the interface and surface charge density. The integration over quadrilateral elements during the discretization is carried out by a linear isoparametric mapping of each element into a unit rectangle. Using the notations in Fig. 1 the mapping is defined by the following equations

$$
\begin{aligned}
x(\xi, \eta)=x_{1}+\left(x_{2}-x_{1}\right) \xi & +\left(x_{3}-x_{1}\right) \eta \\
& +\left(x_{4}-x_{3}-x_{2}+x_{1}\right) \xi \eta \\
y(\xi, \eta)=y_{1}+\left(y_{2}-y_{1}\right) \xi & +\left(y_{3}-y_{1}\right) \eta \\
& +\left(y_{4}-y_{3}-y_{2}+y_{1}\right) \xi \eta
\end{aligned}
$$

where $x$ and $y$ are the global physical co-ordinates and $\xi$ and $\eta$ are the local unit element co-ordinates. This mapping is also used to calculate the electric field at each element and to check the position of particles in the grid.

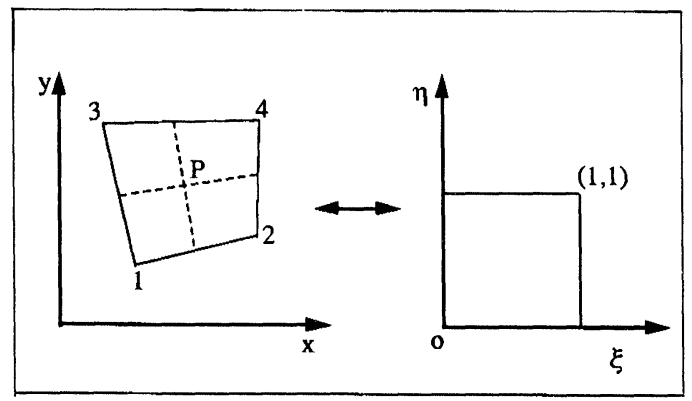

FIGURE 1 Isoparametric transformation: maps the quadrilateral element into a unit square and vice-versa

A complete ensemble Monte Carlo procedure in a single quadrilateral element is the building block of our Monte Carlo module. The motion of each particle is traced, both in momentum space and in real space, under the influence of an averaged uniform electric field. The shape functions are also used in the charge assignment process where the total charge of every super particle is assigned to the four nodes of the quadrilateral element in proportion to the different appropriate areas in the normalised domain and defined by the shape functions.
The position of a particle with reference to a given quadrilateral element is determined using the isoparametric transformation $(x, y) \Rightarrow(\xi, \eta)$. The electric fields are updated at every 1-5 fs depending on the minimum element size and the doping concentration in the heavily doped cap layers of our devices.

Modelling the contacts at the left and right hand edges of the devices poses some difficulties as little is known about the physical penetration of the ohmic contacts through the device layer structure. We have implemented an ohmic contact model that fixes the number of particles in each material layer in the contact region to an initial value obtained by the initial non-linear solution of the Poisson's equation [2].

The MC module was used to study the transient behaviour of FETs. This relies on estimating properly the instantaneous source and drain currents. In theory, the conduction component of the current can be estimated by counting the net number of particles entering or leaving the contacts. The discrete massive super particles makes it difficult to extract reliable time-dependent currents and the counting technique is only useful in evaluating the steady-state currents. In the transient simulations the instantaneous source and drain currents are estimated using a statistical enhancement method [3] which takes into consideration the contribution of all particles in the source and drain regions to the conduction currents. The displacement currents are also evaluated at each time step.

\section{RESULTS AND DISCUSSION}

In this section we demonstrate the capabilities of the Monte Carlo simulation module by example simulations of short recess gate 200nm pseudomorphic HEMT. The layer structure of the recessed p-HEMT is shown in figure (2). Figure (3) shows the I-V characteristics of the $200 \mathrm{~nm}$ gate length p-HEMT obtained from the Monte-Carlo and the Drift-Diffusion modules of $\mathrm{H} 2 \mathrm{~F}$ together with the experimental data. The transient simulations of this device were carried out by first establishing the steady-state condition of operation for $\mathrm{V}_{\mathrm{D}}=1.5 \mathrm{~V}$ and $\mathrm{V}_{\mathrm{G}}=-0.5 \mathrm{~V}$. 


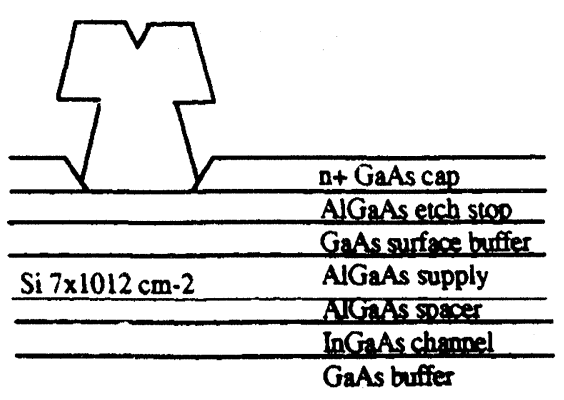

FIGURE 2 Layer structure of a 200nm recess gate p-HEMT

This is then followed by changing the gate voltage to $\mathrm{V}_{\mathrm{G}}=0 \mathrm{~V}$. The evolution of the drain current following the gate perturbation is shown in figure (4) for different cap-layer donor concentrations, viz. $\mathrm{N}=4 * 10^{18}, 2.5 * 10^{18} \& 10^{18} \mathrm{~cm}^{-3}$. Terahertz oscillations are clearly observed in the transient region. The amplitude of the current swings and the frequency of oscillations were found to be independent of the mesh size and the simulation time step. The results shown in figure (4) correspond to a 95X60 mesh and a fieldadjusting time step of $\Delta \mathrm{T}=2.5 \mathrm{fs}$. The oscillations were also found to be independent of the total number

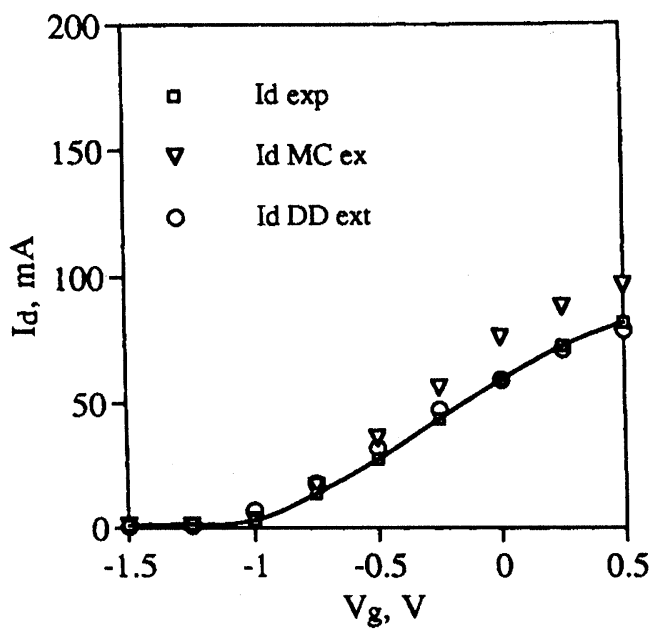

FIGURE $3 \mathrm{I}-\mathrm{V}$ characteristics of the p-HEMT obtained from the Monte-Carlo (MC) and Drift Diffusion (DD) modules of H2F together with the experimental data of super particles employed in the simulation, i.e. independent of the size of the super particle. Zhou and Ferry [5] reported similar oscillations from the simulation of a $25 \mathrm{~nm}$ MESFET employing the quantum moment equations. The Fourier transforms of the drain currents, figure (5), indicate the presence of secondary peaks which may correspond to modes generated at regions of different charge densities and different effective masses. The results presented in this paper and those in $[4 \& 5]$ represent the intrinsic device transient behaviour and do not include the effects of the gate resistance and the contact and external series resistances.

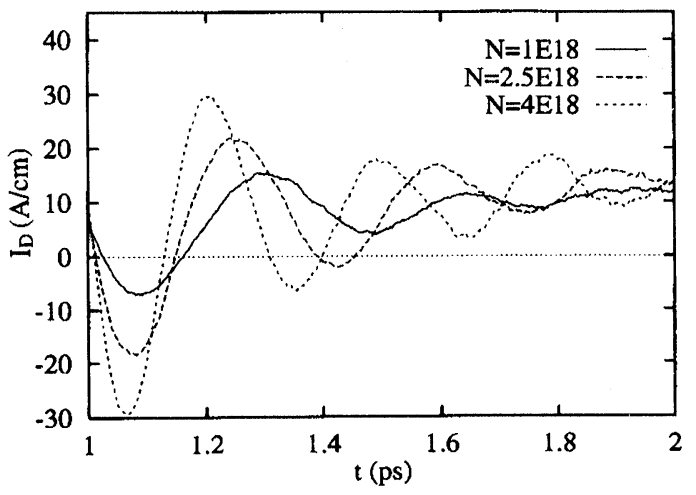

FIGURE 4 Switching behaviour of the pseudomorphic HEMT. $\mathrm{V}_{\mathrm{D}}=1.5 \mathrm{~V}, \mathrm{~V}_{\mathrm{G} 1}=-0.5, \mathrm{~V}_{\mathrm{G} 2}=0 \mathrm{~V}$

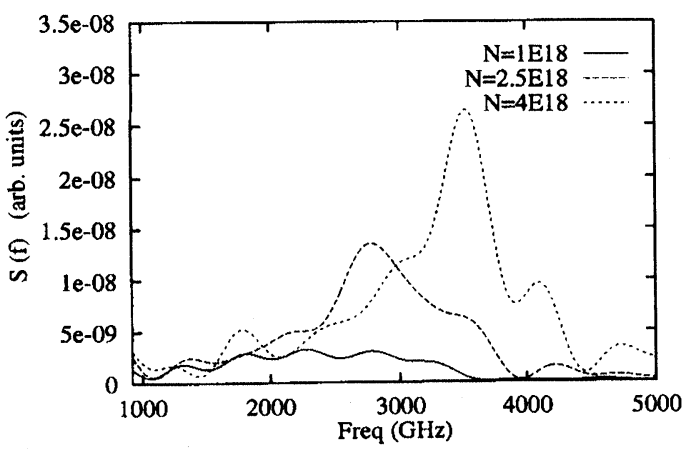

FIGURE 5 Fourier transforms of the transient currents from Figure 4 


\section{CONCLUSION}

In this paper we have described a new Monte Carlo module that is capable of modelling accurately recessed gate MESFETs and HEMTs. An important feature of our particle simulation approach is the use of a quadrilateral Finite-Element discretization scheme to represent the device geometry in the best possible way. The capabilities of our module has been illustrated in example simulations of compound FETs. The transient response of the example p-HEMT showed oscillations that are related to the doping level at the donor layer.

\section{References}

[1] A. Asenov, D. Reid, J. R. Barker, N. Cameron and S. P. Beaumont, in Simulation of Semiconductor Devices and Processes, eds. S. Selberherr, H. Stippel, E. Strasser, 265268, 1993.

[2] S. Babiker, A. Asenov, J. R. Barker and S. P. Beaumont, 'Finite element Monte Carlo simulation of recess gate compound FETs', Solid State Electronics, 39, 629-635, 1996.

[3] T. Gonzalez and D. Pardo, 'Monte-Carlo determination of the intrinsic SSEC of MESFET', IEEE Trans. on Electron Devices, 24 (4), 605-611, 1995.

[4] J. R. Zhou and D. Ferry, 'Simulation of ultra-small GaAs MESFET using quantum Moment equations', IEEE Trans. on Electron Devices, 39 (3), 473-478, 1992.

\section{Biographies}

Dr. Babiker is a researcher at the Nanoelectronics Research Centre (GU). Had his first degree in Electrical Engineering from the University of Khartoum, Sudan 1984, and an MSc from Essex University 1987. His $\mathrm{PhD}$ research was on Single Electronic Devices and Systems. His current research interests include modelling ultra-small devices and parallel algorithms.

Dr. Asenov (GU) had 10 years industrial experience as a head of the Process and Device Modelling Group in IME - Sofia. In 1985 he developed one of the first integrated process and device CMOS simulators IMPEDANCE. In 1989-1991 he was a visiting professor at the Physics Department of TU Munich.
He joined the Department of Electronics and Electrical Engineering at Glasgow University in 1991. As a leader of the Device Modelling Group he has a major contribution in the development of the $2 \mathrm{D}$ and $3 \mathrm{D}$ device simulators and their application in the design of compound FETs, SiGe MOSFETs and IGBTs. He is working also on the design and performance of parallel algorithms.

Prof. Barker (GU) has been a Professor in the Department of Electronics and Electrical Engineering since 1985 . He has a long standing interest in computational methods, device modelling and transport theory. From 1970-78, 1980-85 he was a member of the Theory group in the Dept. of Physics, University of Warwick. During 1978 and 1979 he worked at the IBM T.J. Watson Laboratory, Yorktown Heights, North Texas State University and Colorado State University. From 1987 to 1989 he was academic director of the IBM UK/Glasgow University Kelvin Project on Numerically Intensive Parallel Computing. He is academic director of the Parsytec Parallel Processing Centre at the University of Glasgow.

Prof. Steven Beaumont (GU) was educated at the University of Cambridge and has been with the Department of Electronics and Electrical Engineering at the University of Glasgow since 1978. He became Head of Department in 1995 and he convenes the Nanoelectronics Research Centre's management committee. His research interests lie in the field of nanometre-scale fabrication and its application to electronic and optoelectronic devices. He has over 100 publications on electron beam nanolithography, dry etching, short-gate III-V based transistors, quantum transport devices, the optical properties of quantum dots, and single electron devices. Latterly he has become involved with the issue of manufacturability of mm-wave circuits and the use of nanometre-scale fabrication techniques coupled with technology-based device simulations to forecast performance and yield with the minimum of process iterations. 

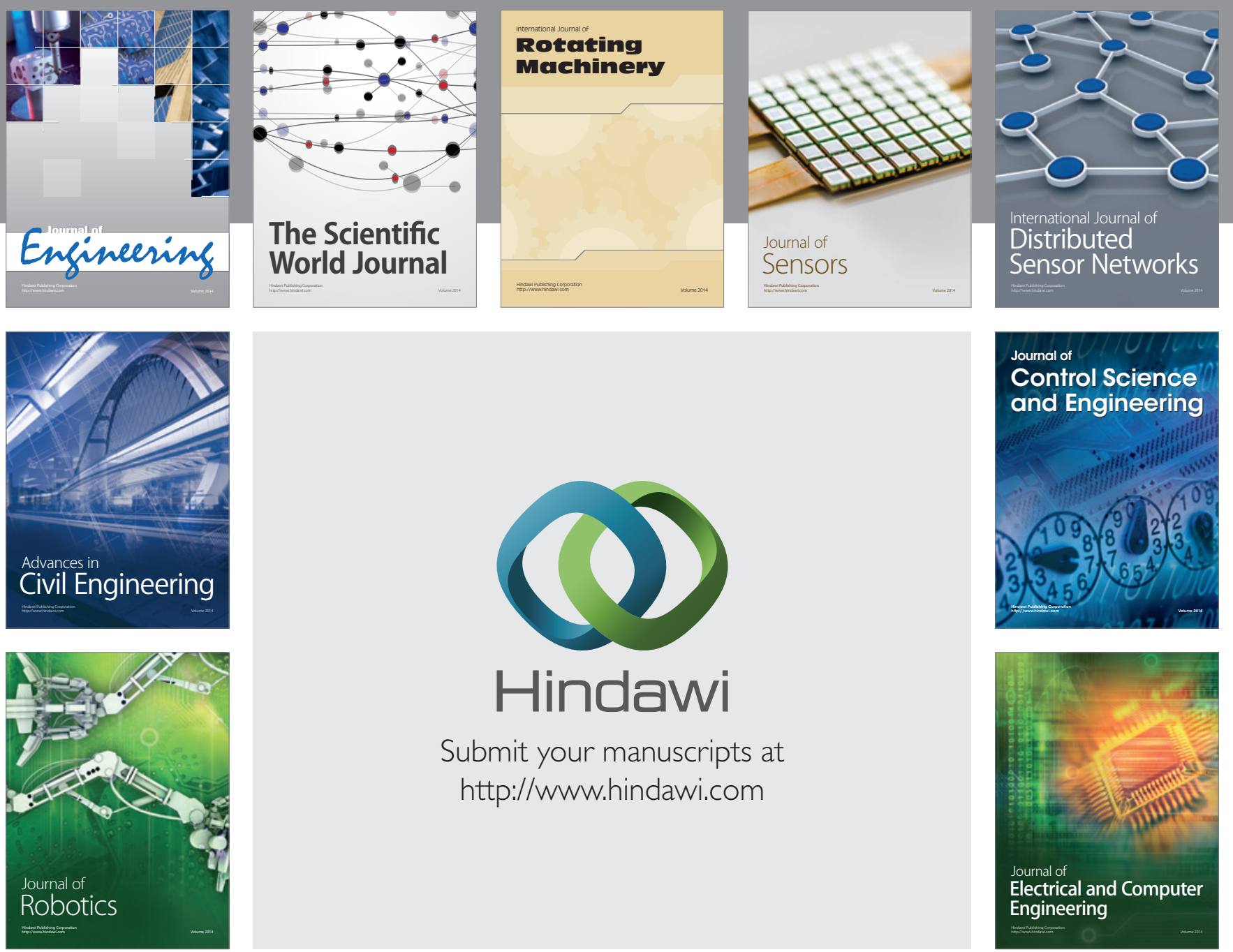

Submit your manuscripts at

http://www.hindawi.com
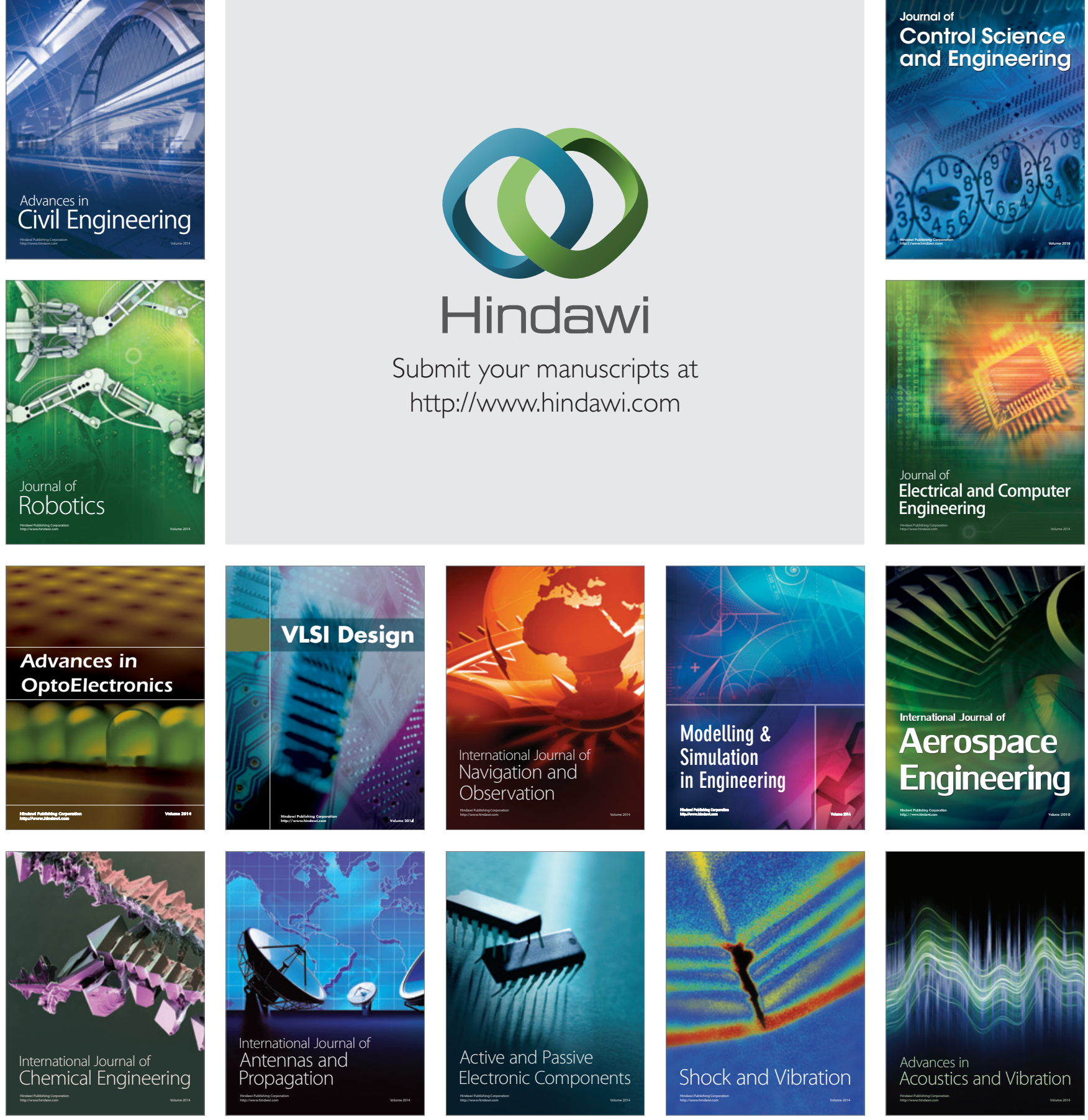